\title{
LEAVING THE BENCH FOR THE CLINIC
}

\section{The increased demands on Clinical Research \\ Associates mean that inexperienced applicants from academia should learn more about the clinical development process.}

Discovering and developing drugs is an expensive business, and by far the most expensive part of this process is testing drugs in humans. According to the Tufts Center for the Study of Drug Development ${ }^{1}$, the average cost of developing new therapeutic compounds approved in the 1990s was US $\$ 802$ million (in year $2000 \$$ ). Clinical development costs, on average US $\$ 467$ million, made up more than half the total cost.

Study delays, such as slow patient recruitment, protocol amendments and review processes, are contributing factors. Every day that a drug is prevented from being on the market means a loss of sales, which in the case of blockbuster drugs can be as much as US $\$ 4-5$ million per day.

So, ensuring that clinical trials run smoothly and efficiently is of the utmost importance for companies. And much of the driving force behind this comes from Clinical Research Associates (CRAs). CRAs supervise, monitor and support the administration and progress of a clinical trial on behalf of a company, which can be either a pharmaceutical company or a contract research organization (CRO).

The role of a CRA is becoming more demanding. Site management is an increasingly challenging task, and CRAs must work much more closely with the site to ensure that regulatory requirements are met. Monitoring has also become more intense to keep in line with The International Conference on Harmonisation of Technical Requirements for Registration of Pharmaceuticals for Human Use (ICH; see BOX 1) and with the various privacy laws, such as the Health Insurance Portability and
Accountability Act of 1996 (HIPAA) in the United States, and the Personal Information Protection and Electronic Documents Act (PIPEDA) in Canada. The European Union's Clinical Trials Directive, which intends to simplify and harmonize the regulation of trials across the European Union and is due to begin on 1 May 2004, will place further demands on CRAs.

It is no surprise, therefore, that most job adverts for CRAs ask for experience. So, what chance does someone from academia have of entering the field? A deep understanding of a particular therapeutic area at the Ph.D. level is still a desirable quality. In-house positions are available, as there is a requirement for experts in the various fields who are able to converse with the physicians running the studies at the clinics and hospitals. The many full-time and parttime Masters courses in clinical research that have sprung up in recent years are attractive, because they save the company from investing time and money in training; however, many companies see such qualifications as more of a bonus than a key factor in hiring. In addition, organizations such as the Institute of Clinical Research (BOX 2) run workshops on becoming a CRA. Experience or knowledge of the pharmaceutical industry and in particular the clinical development process helps. This area is filled with unique terms and initiatives (BOX 1), and doing your homework and making it clear how you can adapt the skills you have developed to this area will help no end in securing a position.

1. DiMasi, J. A., Hansen, R. W. \& Grabowski, H. G. The price of innovation: new estimates of drug development costs. J. Health Econ. 22, 151-185 (2003)

\section{Box 1 | Glossary of clinical trial terms}

Phase I. In clinical development, an experimental drug goes through Phase I-III trials before it can gain approval to be administered to consumers. Phase I trials are an initial assessment of safety, pharmacokinetics and pharmacodynamics in a small number of healthy subjects.

Phase II. Assesses efficacy and safety in a larger number of patients (typically hundreds) with the relevant condition. Often includes pharmacokinetic assessments at various dose ranges to determine optimal dose.

Phase III. Assesses short- and long-term safety and efficacy in a large group of patients (typically hundreds to thousands). Treatment is compared with placebo or competitor drug, allocated to patients by blinded randomization.

The International Conference on Harmonisation of Technical Requirements for Registration of Pharmaceuticals for Human Use (ICH). A single application, called the Common Technical Document (CTD), reduces duplication of testing carried out during the research and development of new treatments in Europe, Japan and the United States - the three biggest pharmaceutical markets.

Good Clinical Practices (GCP). Standard regulations from a regulatory authority for the design, conduct, performance, monitoring, auditing, recording, analysis and reporting of clinical trials.

Source Document Verification (SDV). Verification by the company that the clinical trial data recorded in a trial subject's case report form (CRF) can be supported by the subject's source documents, and that the subject's medical records include all trial-related information that might have an impact on the continued safety, rights and well-being of the subject.

\section{Box 2 | Further information on Clinical Research Associate careers}

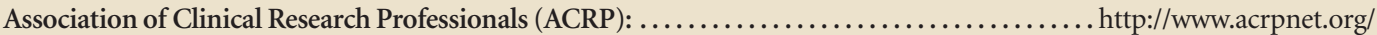

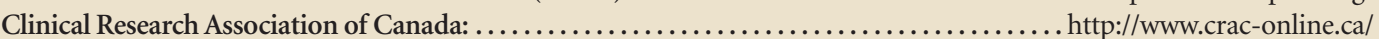

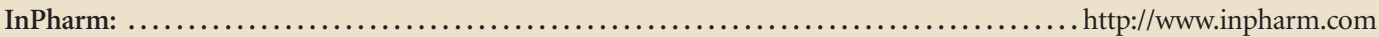

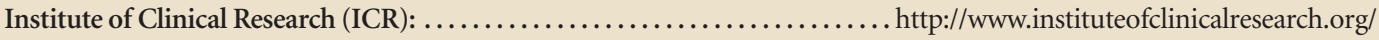

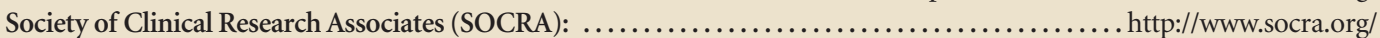

Simon Frantz, Associate Editor (News), Nature Reviews Drug Discovery e-mail:s.frantz@nature.com 